\title{
Fused Multi-Characteristic Validity Index: An Application to Reconstructed Image Evaluation in Electrical Tomography
}

\author{
Shihong Yue * \\ Electric Engineering and Automation, Tianjin University \\ Tianjin Key Laboratory of Process Measurement and Control \\ Tianjin 300072, China \\ E-mail: shyue1999@tju.edu.cn *correspondence author \\ Teresa Wu \\ School of Computing, Informatics, Decision Systems Engineering, Arizona State University \\ Tempe, AZ, 85287, USA \\ Zhiqing Liu \\ Dept. of Logistic Command and Engineering, Naval Univ. of Engineering \\ Tianjin 300452, China \\ Xian Zhao \\ Electric Engineering and Automation, Tianjin University \\ Tianjin 300072, China
}

\begin{abstract}
The quality of reconstructed images is an important and direct criterion to quantitatively evaluate the effectiveness of reconstruction algorithms in electrical tomography (ET). Unfortunately, there lacks of effective and efficient approach to assessing the quality of ET images in literature and practices. Realizing the gap, we recently develop a novel index termed fused Multiple Characteristic Indices (fMCI) ${ }^{1}$ aiming to measure the quality of clustering various data sets including imaging data (e.g., ET). In this paper, we propose a method based on fMCI to quantitatively evaluate the quality of reconstructed images. The method first applies the fast fuzzy c-means algorithm to cluster pixels in the reconstruct image. The fMCI is then applied to evaluate the clustering results and image quality. Simulated data derived from finite element method is used to demonstrate that the proposed method is capable to evaluate the quality of the reconstructed images and the results are consistent with visual perception. In addition, a number of experiments are conducted to demonstrate the applicability and effectiveness of the fMCI based method for image quality assessment.
\end{abstract}

Keywords: Validity index, Electrical tomography, image reconstruction, image evaluation.

\section{Introduction}

Electrical Tomography (ET) has gained considerable interest in both industrial measurement and biomedical engineering ${ }^{2,3}$. Although great progress has been made in improving ET technique in the past two decades, the quality of ET images such as resolution is less satisfactory, mainly due to the known fact that the image reconstruction is an inverse problem with illconditioned property ${ }^{2,3,4}$. Though much work has been done by researchers improve ET image reconstruction, the state-of-art imaging resolution assessment is still limited to single-parameter evaluation of binary images.
To address this issue, researchers have studied the unique characteristics of ET reconstructed images and explored multi-parameter, quantitative evaluation methods. For example, $\mathrm{Woo}^{4}$, Kotre and $\mathrm{Adler}^{3}$ presented the definitions of spatial resolution from different aspects. Wheeler ${ }^{5}$ used parameters related to spatial position and centre resolution to evaluate the spatial resolutions of different areas of the image. Zhao ${ }^{6}$ proposed a multi-parameter method (includes area error and image centre position error) for the electrical capacitance tomography (ECT) image evaluation and a calculation model for the image centre position error. Graham and Adler ${ }^{7-9}$ defined the resolution in terms of fuzzy radius, and adopted the ratio between the radius of 
the interested region and that of the imaging region, the axial error and the image energy to evaluate the quality of reconstructed images. While promising, the research reviewed above suffers from some limitations. First, most evaluation methods require the inputs from user which tend to be subjective. Secondly, the images studied so far have been either binary or grey-scale. The applicability to color image from ET is not explored. Thirdly, though the methods consider multi-parameters, the parameters studied are relatively simple, such as images error resolution, duty ratio ${ }^{2,9}$. Lastly, some methods only take a known mesh instead of the whole image to identify the "optimal" parameter settings during the reconstruction and evaluation ${ }^{7}, 9$. The performance on the whole images thus is questionable. Consequently, it is desirable to develop a method to comprehensively and objectively evaluate reconstructed images in both grey and color scale. The foremost requirement for such method is a powerful assessment index which can truthfully measure the image quality. Considering the image reconstruction is a procedure to assign each pixel to a color scale thus the whole image is composed of a number of blocks with associated colors, scoping the blocks can be generally treated as a clustering problem. Here we propose a cluster validity index which can be used to quantitatively evaluate the quality of reconstructed images.

Clustering analysis is an unsupervised technique used to assign the objects with similar characteristics to the same group ${ }^{10,11}$. One critical issue in clustering analysis is to validate the clustering results quantitatively, e.g., determining the optimal number of clusters and their optimal clustering configurations. In general, the performance of a clustering method is evaluated through validity indices built upon distance measures. For example, the between-distance function calculates the distance of the objects from two different clusters, and the within-distance function calculates the distance of the objects within the same cluster. By maximizing the between-distance and minimizing the within-distance, an acceptable clustering partition may be reached 12, ${ }^{13}$. However, there exists different formulations of the distance functions (e.g., Euclidean distance) and the applicability of these indices is heavily dependent on how to combine these functions. Over the last few decades, extensive research has been conducted to improve validity indices by integrating various within- and between- distance functions. Instead of improving a single index, we have recently proposed a fused multiple clustering index (fMCI $)^{1}$ to integrate multiple indices for better performance. Specifically, we study the use of the D-S evidence theory ${ }^{14-16}$, a mathematical tool for handling uncertainty and imprecise and incomplete information to fuse multiple complementary characteristics in clustering evaluation. In $\mathrm{fMCI}$, we first introduce the use of weighted average of a group of existing validity indices into the evaluation process. Next, other than commonly used distance measures (e.g., between- and within- distance), we introduce a new two-order difference within distance functions which is applicable to arbitrary structures of data space. We then propose a ratio-type function that consists of neighborhoods of the center and boundary of all clusters. These three characteristics are used as information resources for the D-S theory tool to generate $\mathrm{fMCI}$. The advantages of $\mathrm{AMCI}$ are twofold. It is a generalized validity index, thus is not constrained by the data space nor the clustering algorithms. In addition, fMCI is introduced to assess the clustering outcome considering that the clustering process itself is uncertain, instable and, therefore, incomplete in nature.

To what follows, the overview of the fuzzy clustering validity measures are presented in section 2 . Section 3 discusses the cluster evaluation, clustering characteristic extraction, and fusion of characteristics to estimate the number of clusters. Experiments on simulated data and real data are presented in section 4 to verify the usefulness of our proposed method followed by conclusion drawn in Section 5 .

\section{Related Work}

In this section, we review three commonly used fuzzy validity indices, a fast fuzzy c-means algorithm, the D-S evidence theory tool, and the ET image reconstruction algorithms.

\subsection{Three representative fuzzy validity indices}

Let $X=\left\{x_{1}, x_{2}, \ldots, x_{n}\right\}$ be a dataset in a $d$-dimensional data space, containing $n$ data points distributed in $c$ clusters, $C_{1}, C_{2}, \ldots, C_{c}$. Hereafter, we denote $u_{i j}$ as the membership of $j$-th data point to $i$-cluster in a fuzzy clustering algorithm, $v_{i}$ as clustering prototype of $i$ cluster, for $i=1,2, \ldots, c, j=1,2, \ldots, n$, 


\subsubsection{Bezdek's partition entropy $(P E)$ index}

A commonly accepted validity index in fuzzy clustering is the partition entropy $(\mathrm{PE}){ }^{17}$ proposed by Bezdek,

$$
V_{P E}=-\frac{1}{n} \sum_{i=1}^{c} \sum_{j=1}^{n} u_{i j} \log _{a} u_{i j}
$$

where $a$ is the base of the logarithm. The PE index is a scalar measure of the amount of fuzziness in a given dataset. The index is computed for values of $c$ greater than 1 and its values range in between 0 and $\log _{a} c$. In general, we find an optimal $c *$ by solving $\min _{1<c<n} V_{\mathrm{PE}}$ to produce the best clustering performance for the data set $X$.

\subsubsection{Xie and Beni's $X B$ index}

A validity function proposed by Xie and Beni $(1991)^{13}$ with fuzziness exponent $m=2$ and modified by Pal and Bezdek (1995) was defined by

$$
X B(c)=\frac{\sum_{i=1}^{c} \sum_{j=1}^{n} u_{i j}^{m}\left\|x_{j}-v_{i}\right\|^{2}}{n \min _{i, j}\left\|v_{i}-v_{j}\right\|}=\frac{J_{m}(u, v) / n}{\operatorname{Sep}(v)}
$$

$J_{m}(u, v)$ is a compactness measure and $\operatorname{Sep}(v)$ is a separation measure. In general, an optimal $c^{*}$ is found by solving $\max _{2 \leq C \leq n-1}$ to produce a best clustering performance for the data set $X$.

\subsubsection{Pakhira and Bandyopadhyay's (PB) index}

Pakhira and Bandyopadhyay proposed a validity index called the PB-index ${ }^{19}$. The index was developed for both hard and fuzzy clustering algorithms. Here we only illustrate the fuzzy version of the index:

$$
V_{P B M F}=\left(\frac{1}{c} \times \frac{E_{1}}{J_{m}} \times D_{c}\right)^{2},
$$

where $E_{1}=\sum_{j=1}^{n}\left\|x_{j}-v\right\|$ with $v$ being the centroid of data set, $D_{c}=\sum_{i, j=1}^{c}\left\|v_{j}-v_{i}\right\|$ with $J_{m}$ defined by $J_{m}(U, V)=\sum_{j=1}^{n} \sum_{i=1}^{c}\left(u_{i j}\right)^{m}\left\|x_{j}-v_{i}\right\|$, and $m=1.5$. The optimal number of clusters can be obtained by minimizing Eq. (7) for all possible numbers of clusters.

\subsection{The D-S evidence theory}

In the D-S theory, by representing the uncertainty and the imprecision of knowledge via the notion of evidence, belief can be combined to a single hypothesis (singleton) or a composite hypothesis (union of hypotheses) ${ }^{15}$. A useful operator following the evidence combination rule is introduced to integrate information from different sources. The decision on the optimal hypotheses choice can then be made in a flexible and rational manner.

Let us assume there exists (i) a frame of discernment $X$ consisting of the exhaustive and exclusive hypothesis and (ii) the reference set $2^{X}$ of all the subsets of the elements of $X$. In the D-S evidence theory, a basic probability assignment (BPA) is an elementary mass function: $m: 2^{X} \rightarrow[0,1]$ satisfying: $m(\phi)=1$ and $\sum_{A \subseteq 2^{X}} m(A)=1$. The element of $2^{A}$ that has a non-zero mass value is called focal element, and the union of all the focal elements is called the core of the mass function. In the evidence theory, $m(A)$ is a focal element if $m(A) \neq 0$, and $m(A)$ indicates the certainty degree of $A$. A BPA is characterized by two functions: the belief function $\mathrm{bel}$ and the plausibility function $\mathrm{pl}$. The belief in a subset $A \in 2^{X}$ is the sum of all pieces of the evidences that support $A$, and the plausibility of $A$ is the sum of pieces of evidences not supporting $A$, that is,

$$
\left\{\begin{array}{l}
\operatorname{bel}(A)=\sum_{B \subseteq A} m(B) \\
\operatorname{pl}(A)=\sum_{B \cap A=\phi} m(B)=1-\operatorname{bel}(\bar{A})
\end{array}\right.
$$

Based on these definitions, combination operators can be characterized as

$$
m(A)=\left(1-\sum_{A_{i} \cap B_{j}=C} m\left(A_{i}\right) m\left(B_{j}\right)\right)^{-1} \sum_{A_{i} \cap B_{j}=C} m\left(A_{i}\right) m\left(B_{j}\right)
$$

It is possible to build a unique elementary mass function $m$ from $n$ elementary mass functions $m_{1}, m_{2,} \ldots, m_{n}$ from $n$ distinct and independent sources yet characterized on the same set. The combination rule in the D-S evidence theory consists of calculating:

$$
\operatorname{bel}(A)=\left(\left(\left(\operatorname{bel}_{1} \oplus b e l_{2}\right) \oplus \operatorname{bel}_{3}\right) \oplus \ldots\right) \oplus \operatorname{bel}_{n}
$$

where $\oplus$ denotes the combination operator. A similar equation for the plausibility function is.

$$
p l(A)=\left(\left(\left(b e l_{1} \oplus p l_{2}\right) \oplus p l_{3}\right) \oplus \ldots\right) \oplus p l_{n}
$$

The combination is commutative and associative. The last step is the decision-making process, which is supported by the results provided by the combination rules. After the decision principles are determined, the evidence theory can efficiently fuse a group of information sources in the given frame of discernment.

The D-S evidence theory tool has the ability to handle uncertain, imprecise, and incomplete information $^{1,14}$, and to increase the confidence degree of 
the final decision result. These abilities are desirable for identifying the true number of clusters in this study.

\subsection{ET imaging mechanism and algorithms}

The ET imaging aims to reconstruct an image by the electrical conductivity (permittivity) of materials flowing within the sensor from voltages measured at the periphery of the sensor in response to the injected electrical current. The electrical conductivity $\sigma$ and electrical potential $\varphi$ are governed by the Maxwell equation ${ }^{3,7}$. The ET imaging problem responds to an inverse problem, that is, finding the conductivity distribution $\sigma$ based on the voltage measurements is called the inverse problem. To implement the ET imaging, the investigated area has to be discretized. A common form of the ET inverse problem is computed as

$$
G=S^{-1} U .
$$

where $S^{-1}$ is the inverse matrix of the discrete sensitivity matrix $S$ governed by Maxwell equation, and $G$ is the grey vectors corresponding to the distribution of $\sigma$ of all pixels. The mathematical problem in tomography is an inverse problem which has been demonstrated in [4] and [8] and a unique solution is guaranteed provided that data from an infinite number of views available. However, direct analytical solution for Eq. (8) does not exist since the actual equation is a nonlinear operator with more unknown conductivity values than known voltage measurements. In that context, only approximations of $S^{-1}$ can be found by numerical techniques. The choice of the approximation of $S^{-1}$ in Eq. (8) leads to different image reconstruction algorithms. The simplest one is to use the transpose of $S$, which corresponds to the linear back projection (LBP) ${ }^{20}$ and Landweber method ${ }^{21}$, Eq. (8) is then turned to

$$
\hat{G}=\arg \min _{G}\left\|U_{M}-S^{T} G\right\|_{2}^{2}
$$

In the LBP algorithm the conductivity distribution is assumed to comprise a number of discrete regions within the measurement space such that the conductivity within each region is constant. According to Eq.(6),

$$
S^{-1}=S^{T} \lambda / S^{T} U_{\lambda}, \quad \text { s.t. } U_{\lambda}=[1,1, \ldots, 1]
$$

Eq.(10) shows that the grey values of any pixel is calculated using a weighted form, and $\lambda$ is a unit vector in the algorithm.

The Landweber iteration method was originally designed for solving the classical ill-posed problem using the strategy that is similar to the gradient descending algorithm in the optimization process by the following equation:

$$
\hat{G}_{k+1}=\hat{G}_{k}-\alpha S^{T}\left(S \hat{G}_{k}-\lambda\right)
$$

where the constant $\alpha$ is known as the gain factor and is used to control the convergence rate. As the iterative process described by Eq. (9) proceeds, the norm of the capacitance residual will be minimized.

Regularization techniques have been adopted in order to stabilize the inversion. They are used to determine a set of solutions by using prior constraint information and select an optimum one. Tikhonov method $^{22}$ is one of the most popular regularization tools for solving ill posed inverse problems. The reconstruction is commonly stabilized using the Tikhonov regularization by minimization of the follow function:

$$
J(g)=\|S g-\lambda\|^{2} / 2+\mu R(g)
$$

where $R(g)$ is the regularization function, and $\mu$ is the regularization parameter. The function is often expressed in $\mathrm{L}^{2}$ form as:

$$
R(g)=\|L(g-\bar{g})\|^{2}
$$

where $L$ is an appropriate regularization matrix and $\bar{g}$ is a prior estimate of the permittivity distribution. In practice, it is difficult to obtain $\bar{g}$ when prior information is not available. In general, choosing $\bar{g}$ as zero and $L$ as an identity matrix $I$ give the standard Tikhonov regular.

After reviewing the indices and D-S theory, we will introduce $\mathrm{fMCI}$, a novel index based on fusing multiple characteristics and use of $\mathrm{fMCI}$ for imaging quality evaluation in the next section.

\section{3. fMCI for the image evaluation of electrical tomography}

\subsection{Characteristic extraction to general clustering evaluation}

The first characteristic studied is a within-cluster distance function. Most existing validity indices consist of the within- and between- cluster distance functions. A combined structure of both within- and betweencluster distance functions will greatly impact the effectiveness of these validity indices. Usually the within-cluster distance function is monotonically decreasing as the number of cluster $(N C)$ increases. 
We have previously demonstrated ${ }^{1}$ that the extended two-order difference of within-cluster distance function can help locate the elbow position as the reference curves in the gap statistic index ${ }^{1}$. The extended twoorder difference is defined as

$T(N C)=\max _{N C, N C^{\prime}, N C^{\prime \prime} \in A, N C^{\prime}<N C<N C^{\prime \prime}}\left(W_{N C^{\prime}}+W_{N C^{\prime \prime}}-2 W_{N C}\right)$,

where $A$ is a set of consecutive values of $N C$, and this set contains all possible number of clusters. When $N C C^{\prime}=N C-1, N C{ }^{\prime \prime}=N C+1, T(N C)$ reduces to the standard two-order difference. In light of Eq.(14) the elbow point can be found when $|A|=11$. After applying Eq.(14) to four variants of the set Satimage in UCI, we have found ${ }^{1}$ that each of their curves of $T(N C)$ is divided into the two sections located above and below the average of optimums, respectively. The real $N C$ is close to or contained in those sections. Therefore, a proper withincluster distance function can determine the real $N C$ to some extent. Since $T(N C)$ does not involve the integration of within-cluster and between-cluster distance functions, $T(N C)$ is a more independent measure compared to the existing validity index.

The second characteristic we consider is a measure termed $S(N C)$ that consists of the ratio between average center density $C D(N C)$ and average boundary density $B D(N C)$ of all clusters under the calculated $N C$. For example, $C D(N C)$ and $B D(N C)$ in DBSCAN algorithm ${ }^{10}$ may be calculated by the average data number across all $r$-neighborhoods of core data and across all $r$ neighborhoods of boundary data ${ }^{12,13}$, respectively, where the $r$-neighborhood is a set of data that is centered in the related data objects with radius of $r$; $C D(N C)$ in the most used $k$-means algorithm ${ }^{10}$ is the average number of data in the $r$-neighborhood of any clustering prototype, while $B D(N C)$ is the average number of data that fall into the $r$-neighborhood of the middle point between any pair of clustering prototypes. To locate the true $N C^{*}$, we define

$$
S\left(N C^{*}\right)=\max _{N C} C D(N C) / B D(N C)
$$

That is, a true $N C$ tends to group the data to the center of the clusters and scatter the data to the boundary. We had tested a dataset of 20 clusters in which some clusters are partially overlapped as an example ${ }^{1}$. In using $\mathrm{CM}$ algorithm, when $N C$ is less than 20 , some $r$ neighborhoods of clustering prototype are located in the overlaps of the in-between clusters that contain few data points due to the effect to minimize the objective function of the $\mathrm{CM}$ algorithm. Hence, $C D(N C)$ is less than the value at $N C=N C$ optimal. If $N C$ is larger than 20, $B D(N C)$ in $S(N C)$ increases since some neighborhoods of middle points fall into high-populated data areas. We conclude $S(N C)$ can attain its maximum in the vicinity of $N C$ optimal. The $S(N C)$ in fact generalizes the notation of the partitioning index we previously proposed. Consequently, $S(N C)$ may better predict the true $N C$ independently.

In summary, we propose two general characteristics in clustering evaluation: one is based on an independent within-cluster distance function and the other consists of a neighborhood based function. Integrating these characteristics and available existing validity indices is a challenging task. The D-S evident theory is proposed to accomplish this task.

\subsection{Proposed Index: Fused Multiple Characteristic Index ( $\mathrm{FMCI})$}

In fMCI, the first information source $\left(V_{1}\right)$ is from a group of indices. For any given dataset, if a group of validity indices $f_{11}, f_{12}, \ldots f_{1 \mathrm{~m}}$ is applicable, let us first combine the group of validity indices to construct a characteristic for $A \in 2^{X}$, termed $V_{1}$ by a weighting sum of values of the group of indices:

$$
V_{1}(A)=\mathrm{w}_{1} f_{11}(A)+\mathrm{w}_{2} f_{12}(A)+\ldots+\mathrm{w}_{\mathrm{m}} f_{1 \mathrm{~m}}(A),
$$

where $w_{\mathrm{j}}$ is the weight to represent the importance of $f_{j}(A)$ for all $j, j=1,2, . ., m$. The weighting value is characterized as

$$
w_{j}=m_{j}(A) /\left(\sum_{j=1}^{m} \sum_{A \in 2^{X}} m_{j}(A)\right) /|A|, j=1,2, \ldots, m
$$

where $\sum_{j=1}^{m} w_{j}=1$. If $w_{j}$ is greater (less) than 1 , the impact of $f_{1 j}$ increases (decreases). Specially, if there are two or more sets of consecutive numbers of clusters above the average line, the calculated weighting value of Eq.(16) for any $A$ must decrease since these sets have to share the value 1 according to the definition of the mass function. Inversely, if there is a dominant set of number of clusters above average of line, the value of Eq.(16) must be 1. Thus Eq.(17) can automatically increase and reduce the importance of each index in the group of indices. One criticism of the D-S evident theory is that Eq. (5) may cause a most unreasonable case called "rejected by one vote" for a given hypothesis. Here, however, the formulation of Eq. (16) 
ensures the true $N C$ will not be directly rejected if it is rejected by one index, but suggested by other validity indices.

Let the second information source $\left(V_{2}\right)$ be $T(N C)$ and the third information source $\left(V_{3}\right)$ be $S(N C)$. Assume $X$ represents a frame of discernment consisting of a set of values of $N C$; for any $A \in 2^{\mathrm{X}}$, the focal element in the $\mathrm{D}-\mathrm{S}$ evidence theory is computed as

$f_{i}(A)=\left\{\begin{array}{l}\sum_{N C \in A}\left(F_{i}(N C)-\operatorname{AverF}_{i}(N C)\right) \\ \quad \text { if } F_{i}(N C) \geq \operatorname{AverF}_{i}(N C) \text { for all } N C \in A,(18, \quad \text { otherwise. }\end{array}\right.$

where $\operatorname{Avg} F(N C)$ is the average of all values of $F_{\mathrm{i}}(N C)$ $(i=1,2,3)$ for all values of $N C$. Since the values of different validity indices in Eq.(18) may have different orders of magnitude, using a weighting sum approach to combine a group of validity indices should be normalized. The mass functions in the D-S evidence theory are normalized as

$$
m_{i}(A)=f_{i}(A) / \bar{f}_{i}, \quad \text { for all } A \in 2^{A}, i=1,2,3
$$

where $\tilde{f}_{i}$ is the average of all values of $f_{i}(A)$.

After fusing the above three characteristics by the D-S evidence theory, the decision-making rule is that $A_{1}$, $A_{2} \in 2^{A}$ which satisfies

$$
\begin{aligned}
& m\left(A_{1}\right)=\max \left\{m\left(A_{i}\right), A_{i} \subset U\right\}, \\
& m\left(A_{2}\right)=\max \left\{m\left(A_{i}\right), A_{i} \subset U \text { and } A_{i} \neq A_{1}\right\}
\end{aligned}
$$

If there exists

$$
\left\{\begin{array}{l}
m\left(A_{1}\right)-m\left(A_{2}\right)>\varepsilon_{1} \\
m(X)<\varepsilon_{2}, \\
m\left(A_{1}\right)>m(X)
\end{array}\right.
$$

then $A_{1}$ is the decision-making result, where $\varepsilon_{1}$ and $\varepsilon_{2}$ are pre-established threshold. In this paper we set $\varepsilon_{1}=$ $\varepsilon_{2}=0.05$.

\subsection{Fast fuzzy c-means algorithm to image clustering}

In the context of image clustering, a fast version of the FCM (f-FCM) algorithm can be used ${ }^{23}$. The algorithm is based on a 1-dimensional attribute such as the grey level. Let HS be the histogram of image of $L$ levels, where $L$ is the number of grey levels. Each pixel has a feature that lies in the discrete set

$$
X=\{0,1, \ldots, L-1\}
$$

In this case, each element of the data set represents a grey level value, and we can then use $u_{i l}=u_{i}(l)$ to expresses the membership degree of the $l$-th grey level for the $i$-th cluster. The objective function in the f-FCM can be rewritten as:

$$
J_{m}(U, V, L)=\sum_{l=0}^{L-1} \sum_{i=1}^{C}\left(u_{i l}\right)^{m} \cdot H(l) \cdot\left(l-v_{i}\right)^{2}
$$

where $u_{i l}=u_{i}(l)$ represents the membership degree of grey level $l$ to cluster $i, i=1,2, . ., c$. Thus, the computation of membership degrees of $H(l)$ pixels is reduced to that of a pixel with $l$ as the grey level value. Let $E=\sum_{i=1}^{C} \sum_{l=0}^{L-1}\left|u_{i l}-u_{i l}\right|$. The algorithm first selects the number of clusters $C$, and updates the partition matrix $U$ according to

$$
v_{i}=\sum_{l=0}^{L-1}\left(u_{i l}\right)^{m} \cdot H(l) \cdot l / \sum_{l=0}^{L-1}\left(u_{i l}\right)^{m}
$$

for $i=1,2, \ldots, C$, and

$$
u_{i j}=\left[\sum_{i=1}^{c}\left(\frac{l-v_{i}}{l-v_{j}}\right)^{1 /(m-2)}\right]^{-1}
$$

for $i=1,2, \ldots, C ; l=1,2, \ldots, L$. When $E$ is smaller than a user-specified threshold, the algorithm stops.

\subsection{Evaluation of ET imaging quality}

The f-FCM algorithm is used to cluster all pixels in any ET image that is reconstructed by the three most used imaging algorithms: LBP, Tikhonov and Landweber algorithms respectively, available in our group in Tianjin Key Laboratory of Process Measurement and Control, Tianjin University ${ }^{2,12}$. For any image, each dominant individual color corresponds to a determined grey degree. After applying the f-FCM, these pixels with the same grey degree consist of a cluster. Ideally, the number of clusters in any reconstructed image is identical to the real number of clusters, but this often is impossible since the limited natural space resolution of existing ET imaging techniques ${ }^{5,6}$. Instead, a good reconstructed ET image necessarily has the number of dominant colors that is as close to the real one as possible. Usually, there are a prior number of clusters predefined for any investigated materials since the components of these materials can be known in advance. The ET imaging technique aims to find the distributions of these materials in pipe or vessel. The proposed fused Multiple Characteristic Index (fMCI) can then be 
applied to evaluate the quality of these reconstructed ET images based on two criteria: Accuracy and robustness measure. The Accuracy is measured by the difference between the estimated number of clusters by the fMCI index and the actual number of clusters, and the robustness measure is defined as the difference between the optimal and the second optimal number of clusters for any validity index. The larger the values of the robustness measure are, the stronger the representation for the optimal number of clusters will be. Consequently, the accuracy and robustness measure together can evaluate how well any reconstructed ET image is for a given dataset, comparing to the three fuzzy clustering validity indices PB, PE, and XB discussed in Section 2.

Fig. 1 is a flowchart for our proposed method.

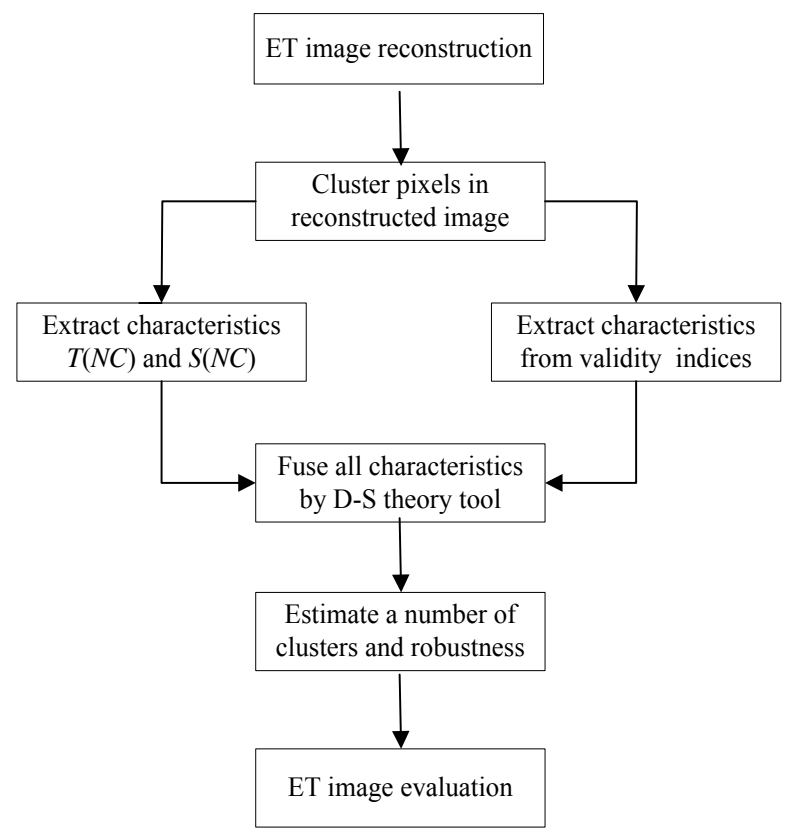

Fig.1. Flowchart of the evaluation processes of the new proposed validity indices for the reconstructed images.

\section{Experiments}

Three artificial and an ET reconstructed image are used to test the effectiveness of the fMCI as follows.

\subsection{Test on three synthetic datasets}

Simulation experiments are performed in the software Comsol $6.7^{2}$. We generate three frames of artificial images with size $256 \times 256$, as shown in Fig. 2. These data (voltages) hidden in these original images are used to reconstruct the ET images by use of the above three ET imaging algorithms, LBP, Tikhonov and Landweber. These reconstructed images and are shown in Table 1.

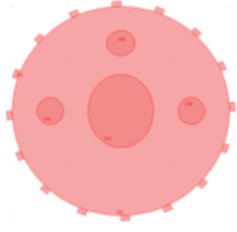

(a)

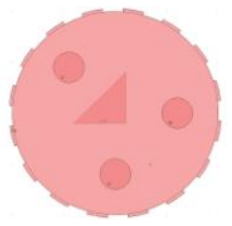

(b)

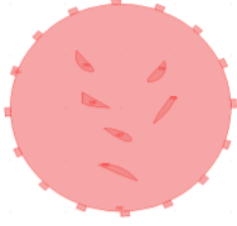

(c)
Fig. 2. Three original artificial images. (a) A big and three small circles with the same permittivity. (b) A triangle and three circles with different permittivity. (c) Six peppers with the same permittivity.

Table 1. Reconstructed images and their evaluations

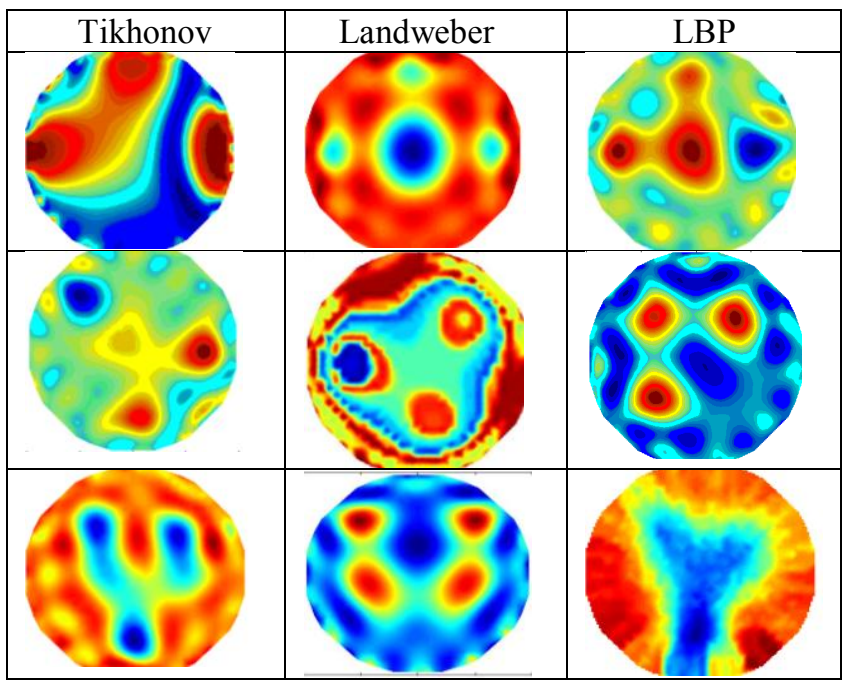

In Fig.2(a) there are three small circles of radius 23 each, and a large circle of radius 60 in the first frame of image, respectively. The pixels of these three small circles have the same grey value 120 and the large circle take grey value 160 , and the background pixels take grey in the value 70 . Thus the number of clusters in the set of vectors from all circles and backgrounds in the fFCM algorithm should be chosen as 3. In Fig.2 (b) the pixels of these three small circles take grey values in the values, 120, 140, 160, and large circle 95, respectively. The background pixels take values in the value 70 . Thus the number of clusters in the set of vectors from all circles and backgrounds in the f--FCM algorithm should be chosen as 5. In Fig.2(c) the third frame of image consists of six irregularly shaped peppers. These pixels of peppers take the same grey values 110 respectively 
while the background pixels take values in the value 95 . Thus the number of clusters in the set of vectors from all peppers and backgrounds should be chosen as 2 . The true numbers of clusters in the three original datasets are used to compute the accuracy and robustness of these images by the fMCI index in the evaluation process.

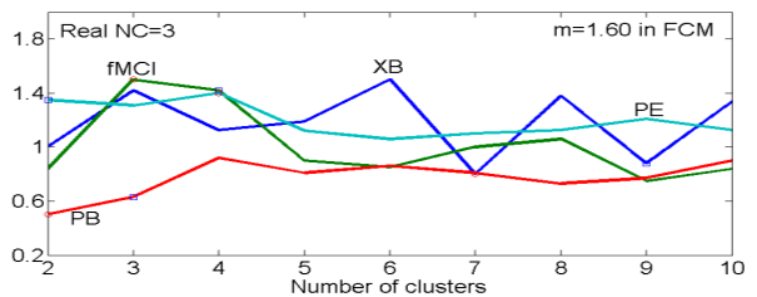

(a)

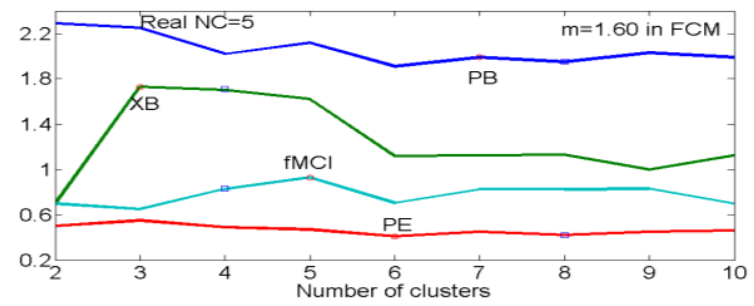

(b)

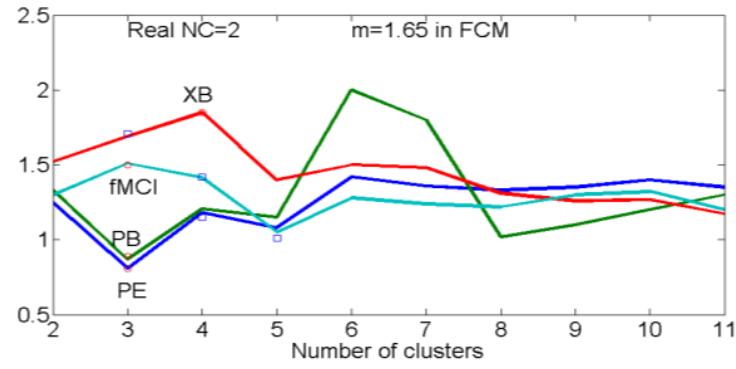

(c)

Fig.3. Comparison of fMCI and the three fuzzy indices $\mathrm{PB}$, $\mathrm{XB}$, and $\mathrm{PE}$ in dataset 1-3 that responds to (a)-(c), where the circle symbol "○" stands for the optimal value of any validity index, and the square symbol " $\square$ " for the second optimal value, the difference between these two symbols represent the robustness of the fMCI.

Table 1 shows these reconstructed images under the three reconstructed ET algorithms, where each row responds to the same original image and thus to the same pixel dataset. Fig. 3 shows the estimated number of clusters by all validity indices. As opposed to the original image, the accuracy and robustness of the fMCI index are the highest. The reconstructed images that the optimal values of the fCMI index points to have the highest space resolution since they are more consistent to the original image than the other images. The boundary of each cluster in these images is tidy and clear. While, the three fused fuzzy validity indices cannot accurately estimate the real number of clusters number of either in accuracy or in robustness measure. The estimated number of cluster by the three indices may be far from the number of dominant colors. Therefore, these fuzzy validity indices themselves may not efficiently evaluate the quality of these reconstructed ET images.

\subsection{Test on image reconstruction for a man lung}

The test results from the diagnose process of a man lung, and experimental data is taken from 32 electrodes around the chest of the man (see Fig.4 (a)). At the same time, the scanned XCT image of the man by using Xray inversion recovery pulse sequences is available to severe as the reference image (see Fig.4 (b)).

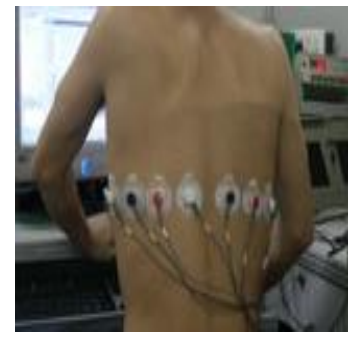

(a)

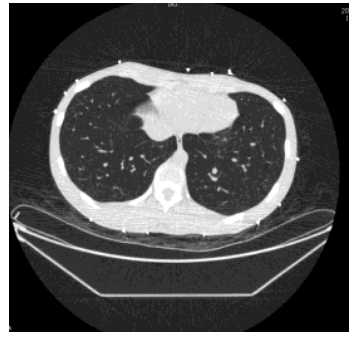

(b)
Fig.4. Experiments tested in a man lung. (a) Data acquisition electrodes. (b) Reconstructed XCT image.

The cross-section is partitioned into 900 units (pixels) for image reconstruction such that it is possible to represent the details of the soft tissues of the man lung in the reconstructed ERT image. We reconstruct the image of the man lung by the LBP, Tikhonov, and Landweber algorithms based on these measured data (voltages) by 32 electrodes. These reconstructed images are shown in Table 2. Usually, the XCT image can reflect the bone skeleton of any man chest well, while the ERT can better reflect the structure of soft tissues to some extent. The man lung approximately contains five kinds of tissues according to rough conductivity differences, thus the actual number of clusters is taken 
as 5. After the f-FCM is applied to cluster pixels in any reconstructed image, the fMCI index is used to automatically to estimate the actual number of clusters for each reconstructed image along a group of consecutive numbers of clusters, comparing with the three fuzzy clustering validity indices $\mathrm{PB}, \mathrm{PE}$, and $\mathrm{XB}$ listed in Section 2.

Table 2. Reconstructed images and their evaluations

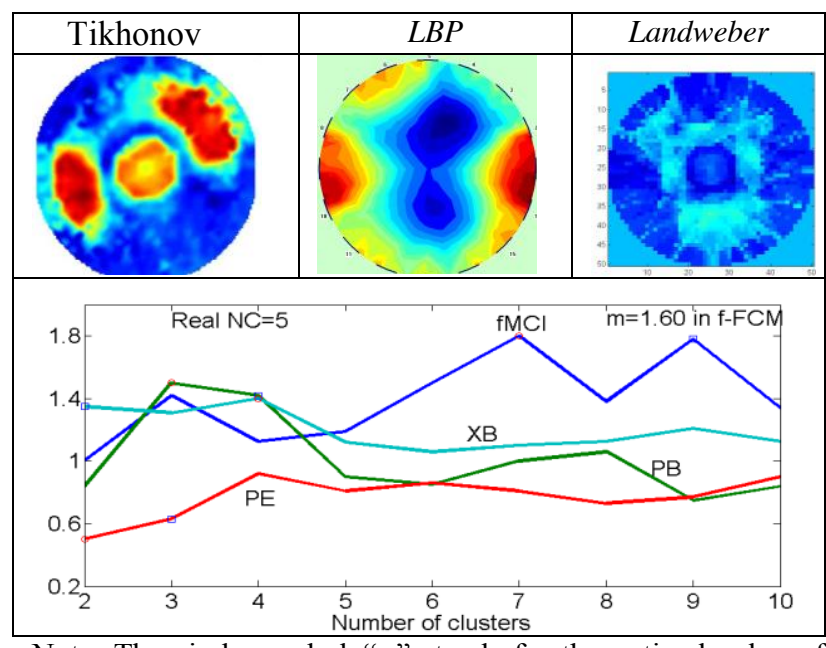

Note: The circle symbol "०" stands for the optimal value of any validity index, and the square symbol " $\square$ " for the second optimal value, the difference between these two symbols represent the robustness of the $\mathrm{fMCI}$.

Table 2 shows that the reconstructed images by the Tikhonov algorithm are the best where these tissue details of a man lung can nearly be identified, and those by LBP algorithm is the worst. These results are consistent with most observations in literature ${ }^{21,23}$. The reconstructed ET image by the Landweber algorithm seems to clearer than the LBP algorithm where boundaries of different clusters look tidier. In the sense of the statistical number of correct partitioned pixels, we have found that $38.17 \%$ and $30.5 \%$ of pixels have been mis-segmented in the classical LBP and Landweber algorithms, but only $20.36 \%$ of pixels are missegmented by the Tikhonov algorithm. This demonstrates that the Tikhonov algorithm is the most acceptable in the three algorithms. Accordingly, the estimated number of clusters by the fMCI index is the closest to the real one by the accuracy and robustness measure. In comparison with the X-ray image, the image by the LBP algorithm can be seen that these clusters seem unclearly expressed since the clusters contain too much tail traces.

Although the optimal values of the PE and PB indices can estimate the correct number of clusters, its robustness measure underperform than the $\mathrm{fMCI}$ index. Therefore, after these individual fuzzy validity indices are fused into a more general index, fMCI, the generalization can be improved.

In addition, the fuzziness exponent of a fuzzy clustering algorithm including the f-FCM index usually may affect the clustering results in a given dataset. In our research we have found that when the values of the fuzziness exponent are generally taken 1.7, the evaluation results of the f-FCM index is thus most acceptable.

\section{Conclusion}

In general, the existing validity indices suffer from the problems of uncertainty, instability, and incompleteness in clustering evaluations ${ }^{24,25}$. In addition, there is lack of a generalized index not bounded to the clustering algorithm and the structure of the data space. To address these issues, we have proposed a novel approach by using the D-S evidence theory to introduce a generalized index by combining multiple aspects from the clustering process. To accomplish this, we first consider combining the three existing fuzzy clustering indices as one information source. Two other functions independent from applied clustering algorithms and structure of data space are introduced as additional information sources for the D-S theory. A fused multiple characteristics index (fMCI) is introduced by fusing the three information sources. Experimental results indicate that the fMCI can generally handle uncertainty, imprecision, and instability issues in clustering evaluation and efficiently evaluate the clustering outcomes of any clustering algorithm and any structure of data space. Essentially, these experimental results provide evidence that the fMCI index obtains a favorable evaluation effect for the reconstructed ET images.

Though the evaluation method presented here is promising and expected to facilitate further research in electrical tomography, there are several directions in 
which the work may be extended. For example, how to effectively define the optimal mass functions for the fMCI to further improve the results remains unanswered. Second, how to choose the combination rules in the DSevidence theory for better estimation of the true number of clusters needs to be explored. These are our research efforts for the future.

\section{Acknowledgements}

This work was supported by the National Science Foundation of China under Grant No. 61174014, 60772080, 60532020 and the Tianjin Science Foundation of China under Grant No. 08JCYBJC13800.

\section{References}

1. S. Yue, T.Wu, Y.Wang, K.Zhang, and W.Liu, Dampster-Shafer Evidence Theory Based MultiCharacteristics Fusion for Clustering Evaluation, Lec. Note Art. Inter. 6401(2010) 499-519.

2. C. Wang, X. Qian, Y. Yan, et al, An Evaluation Method for Reconstructed Images in Electrical Tomography, in IEEE Int. Instr. Mea. Tech. Conf., pp. 12-15, 2008.

3. E. J. Woo, P. Hua. J. G. Webster and W. J. Tompkins. A robust image reconstruction algorithm and its parallel implementation in electrical impedance tomography. IEEE Trans. Med. Imaging, 12 (3) (1993)137-144.

4. C.Kotre, EIT image reconstruction using sensitivity weighted filtered back projection, Physiol. Meas. 15(suppl A) (1994)125-136.

5. J. Wheeler, W. Wang and M. Tang, A comparison of methods for measurement of spatial resolution in two-dimensional circular EIT images, Physiol. Meas. 16(23) (2002)169-176.

6. J. Zhao, W. Fu and Q. Hu, Investigation of evaluating method for reconstructed image quality of electrical capacitance tomography system, $J$. Guangxi University 28(6)(2003)61-64.

7. A. Adler and R. Guardo, Electrical impedance tomography: regularized imaging and contrast detection, IEEE Trans. Med. Imaging 15(3)(1996) 170-179.

8. B. Graham and A. Adler, A nodal jacobian inverse solver for reduced complexity EIT Reconstruction, Int. J. Infor. Sys. Sci. 13 (2) (2006) 453-468.

9. H. Sheikh and A. Bovik, Image information and visual quality, IEEE Trans. Image Processing 2(15) (2006) 430-444.

10. U. Maulik and S. Bandyopadhyay, Performance evaluation of some clustering algorithms and validity indices, IEEE Trans. Pattern Anal. Mach. Intel. 24 (12) (2002)1650-1654.
11. M. K. Pakhira, S. Bandyopadhyay and U. Maulik, A study of some fuzzy cluster validity indices, genetic clustering and application to pixel classification, Fuzzy Sets Syst. 155(3) (2005)191214.

12. D. L. Davies and D. W. Bouldin, A cluster separation measure, IEEE Trans. Pattern Anal. Machine Intell. 1(4)(1979) 224-227.

13. X.L. Xie and G. Beni, A validity measure for fuzzy clustering, IEEE Trans. Pattern Anal. Mach. Intel. 13(8)(1991)841-847.

14. L. Ana, N. Fred, and A. K. Jain, Combining multiple Clusterings using evidence accumulation, IEEE Trans. Patt. Anal. Mach. Intell. 27(6)(2005) 835-851.

15. W. Zhang, Y. Lee, The uncertainty of reasoning principles. (Xi'an Jiaotong University Press, 1999), pp.223-263.

16. F. Cuzzolin, A geometric approach to the theory of evidence, IEEE Trans. SMC-C. 38(4) (2008) 522534.

17. J. C. Bezdek, Pattern Recognition with fuzzy objective function algorithms, Plenum Press, (New York, 1981), pp.12-27.

18. M. Kim, and R. S. Ramakrishna, New indices for cluster validity assessment, Pattern. Recognition Letter 26(6) (2005)2353-2363.

19. M. K. Pakhira, S. Bandyopadhyay, U. Maulik, Validity index for crisp and fuzzy clusters, Pattern Recognition 37(3)(2004)487-501.

20. F. Santosa and M. Vogelius, A back projection algorithm for electrical impedance imaging, SIAM J. Appl. Math. 50(1990) 216-243.

21. W. Q. Yang, D. M. Spink, T. A. York and H. McCann, An image-reconstruction algorithm based on Landweber's iteration method for electricalcapacitance tomography Meas. Sci. Technol. 10 (1999) 1065-1069.

22. M. Vauhkonen, D. Vadasz, P. A. Karjalainen, E. Somersalo and J. P. Kaipio, Tikhonov regularization and prior information in electrical impedance tomography, IEEE Trans. Med. Imaging 17(1998) 285-289.

23. A. Boudraa and P. Clarysse, Fast fuzzy grey level image segmentation method, Medical Biological Engineering Computing, 35(2)(1997)807-817.

24. S. Yue; J. Wang, T. Gao, An unsupervised grid-based approach for clustering analysis, Science ChinaInformation Science, 53(7)(2010)1345-1357.

25. G. Wei, X. Zhao and R.Lin, Some induced aggregating operators with fuzzy number intuitionitic fuzzy information and their application to group decision making, Int. J. Comp. Intel. Syst., 3(1) (2010) 84-95. 